\title{
ACELERÓMETROS MEMS EN EL DESARROLLO DE POZOS Y CAMPOS PETROLEROS INTELIGENTES
}

\section{MEMS ACCELEROMETERS FOR DEVELOPMENT OF INTELLIGENT WELLS AND OIL FIELDS}

\author{
MSc. Jorge E. Meneses Flórez, Ing. José D. García López. \\ Ing. Diego A. Ferreira Pereira. \\ Universidad Industrial de Santander (UIS), Grupo de Investigación CPS. \\ Ciudad Universitaria, Bucaramanga, Santander, Colombia. \\ Tel.: (+577) 6344000 Ext. 2483/2829. \\ E-mail: jmeneses@uis.edu.co, \{jdanielgl, diegof83\}@ hotmail.com
}

\begin{abstract}
Resumen: Sensores instalados en los pozos permiten un monitoreo continuo de los mismos y de todo un campo productor, pero su costo limita su aplicación en campos maduros, donde la producción es baja y el presupuesto limitado. Sensores tipo MEMS, podrían ser utilizados a una pequeña fracción del costo de otro tipo de sensores. Se muestran los resultados de la utilización del acelerómetro MMA7361L de Freescale $^{\mathrm{TM}}$, con el fin de obtener la posición instantánea de un sistema de bombeo mecánico. La posición instantánea de la barra pulida es una de las variables físicas importantes, para obtener el dinagrama de fondo de pozo, a partir del cual se puede identificar la falla presente en el sistema de bombeo.
\end{abstract}

Palabras clave: Dinagrama, pozo inteligente, bombeo mecánico, acelerómetros mems.

\begin{abstract}
Sensors installed in wells allow continuous monitoring of a single well and a complete producing field, but its cost limits its application in mature fields where production is low and the budget is limited. MEMS type sensors could be used by a fraction of the cost of other types of sensors. It has showed the results when a Freescale $^{\mathrm{TM}}$ MMA7361L accelerometer was used, in order to obtain the instantaneous position of a mechanical pump. The instantaneous position of the polished rod is one of the important physical variables for the dynagraphic chart downhole, from which it is possible to identify a failure in the pumping system.
\end{abstract}

Keywords: Dynagraphic charts, smart well, mechanical pumping, mems accelerometers.

\section{INTRODUCCIÓN}

Pozos y campos inteligentes, son conceptos modernos que conducen al aumento de la producción de un campo petrolero, (de Best, 2012; de Best, 2006). La automatización es la base para que pozos o campos petroleros sean inteligentes. A través de la automatización de los pozos, es posible controlar de forma remota la producción de un campo y garantizar el máximo de producción a un costo mínimo de operación, extendiendo la vida útil del equipo. Ante tantas bondades se ha generado un interés creciente en la industria petrolera de poseer pozos y campos inteligentes, (Moises, 2008).

Automatizar implica el uso de hardware (sensores, pre-actuadores, actuadores, controladores) y de software, (Pari, 2009; Gao, 2007). El alto costo de estos sistemas de automatización, limita 
sustancialmente su masificación, especialmente en los campos petroleros maduros o marginales, cuya longevidad conlleva a la natural disminución de la producción, y por ende se tiene muy poca disponibilidad para inversiones. En Colombia un gran porcentaje de la producción actual, proviene de campos maduros. La necesidad, y por ende la oportunidad, radica en el desarrollo de herramientas (hardware/software) que contribuyan al desarrollo propio del concepto de pozo y campo inteligente, a través de la cual se pueda identificar con precisión y rapidez los problemas (fallas) en un pozo, con el fin de disminuir el riesgo operacional y mejorar la producción.

En el mundo cerca del $90 \%$ de los pozos utilizan algún método de levantamiento artificial. El método más utilizado es el bombeo mecánico (figura 1), el cual utiliza una bomba de desplazamiento positivo ubicada en el fondo del pozo (a miles de metros) que recibe un movimiento lineal alternativo generado por una unidad de bombeo situada en la superficie. La transmisión del movimiento a la bomba se hace mediante la unión de una serie de varillas (sarta) de bombeo.

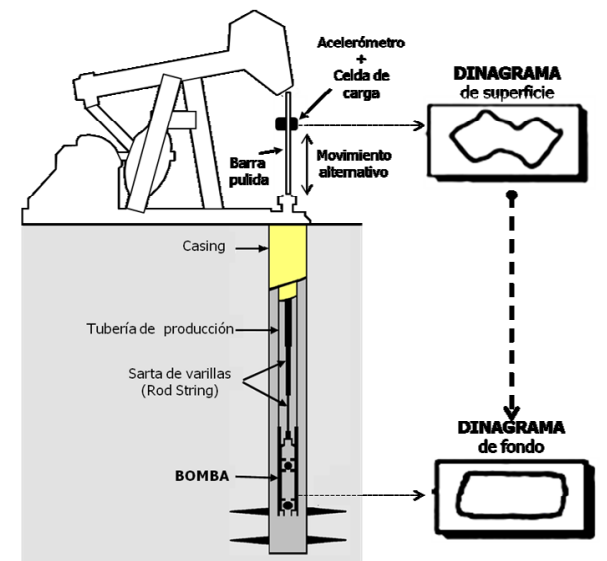

Fig. 1. Sistema de bombeo mecánico y dinagramas.

En el bombeo mecánico, la bomba es el núcleo del sistema, y su desempeño tiene un impacto directo en el nivel de beneficio económico del pozo y del yacimiento de petróleo, (Sánchez, 2007). Es evidente la dificultad para realizar mediciones directamente sobre la bomba, en el fondo del pozo. Entonces, para monitorear su desempeño, un dispositivo denominado dinamómetro de superficie, se une a la barra pulida en la cabeza del pozo. El dinamómetro mide la fuerza (carga) en la barra pulida (en la cabeza del pozo) en función de su posición, para generar una curva (gráfica) que suele denominarse dinagrama de superficie, (McCoy, 1995). El dinagrama es una curva cerrada, en la cual el eje $x$ representa el desplazamiento de la sarta de varillas en cabeza de pozo, y el eje $y$ representa la carga (fuerza) presente para dicha posición, describiendo la relación carga - desplazamiento en un ciclo de bombeo. Modelando el comportamiento del crudo bombeado, y el comportamiento de la sarta de varillas que conecta la superficie con el fondo del pozo, se obtiene el dinagrama de fondo, mediante la aplicación de procesos matemáticos (figura 1). El dinagrama de fondo es la fuente de información fundamental para diagnosticar cualquier falla del sistema de bombeo mecánico.

Como se puede concluir, una de las variables físicas importante para obtener el dinagrama de un pozo, es la posición instantánea de la barra pulida de la unidad de bombeo mecánico. Con esta perspectiva, el trabajo aquí presentado se enfocó en buscar una alternativa económica, evaluando la utilización de acelerómetros de tecnología MEMS, a partir de los cuales, mediante metodologías de cálculo, se obtiene la posición instantánea.

\section{PLATAFORMA DE EXPERIMENTACIÓN}

Para evaluar la aplicación de los acelerómetros tipo mems, se diseñó y desarrolló una plataforma de experimentación (figura 2) basada en: un emulador de un sistema de bombeo mecánico, un sistema de adquisición de datos y software de procesamiento de datos.

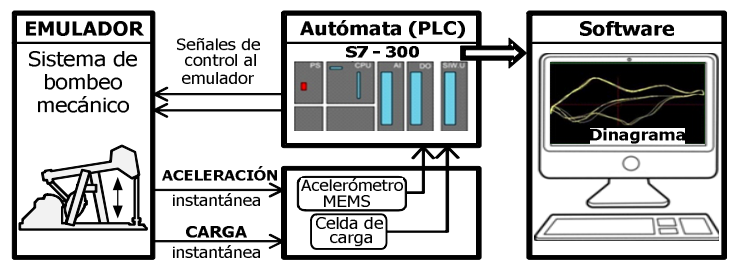

Fig. 2. Plataforma de experimentación.

\subsection{Emulador de un sistema de bombeo mecánico}

Con el fin de obtener dinagramas en tiempo real, se desarrolló un Prototipo Emulador de una Unidad de Levantamiento Artificial (PEULA), ver figura 3. El movimiento alternativo presente en las unidades reales es emulado mediante un mecanismo manivela-biela-corredera, donde el movimiento rotacional del motor se transforma en un movimiento alternativo de traslación de una corredera, emulando así, el movimiento presente en la barra pulida de las unidades mecánicas de bombeo. 


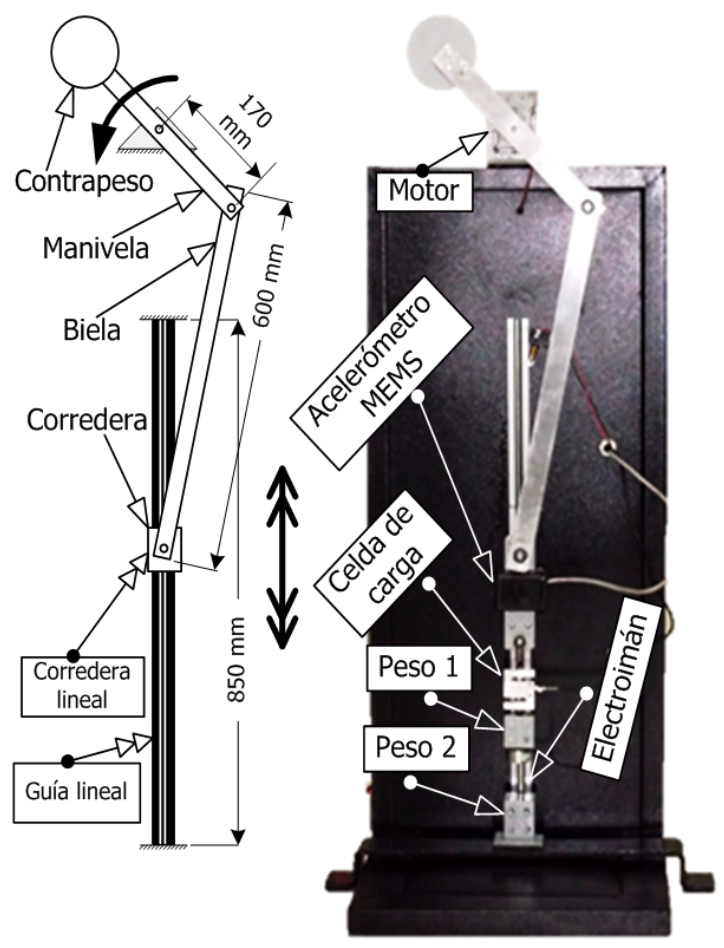

Fig. 3. PEULA.

De la misma forma que el PEULA emula el comportamiento de traslación alternativo, éste debe responder a los comportamientos de carga presentes en las unidades mecánicas de levantamiento artificial. Éstas poseen una sarta de varillas cuyo peso está presente, tanto en la carrera ascendente como en la descendente, el cual fue emulado mediante una carga de peso fijo (peso 1).

La acción de bombeo del fluido, que aparece en la carrera ascendente, fue emulada mediante un peso adicional (peso 2), que es recogido en el punto muerto inferior del mecanismo, mediante la acción de un electroimán.

El peso 2 es soltado en el punto muerto superior, iniciando la carrera descendente. El control de la velocidad del motor de la manivela del PEULA, permite variar el número de carreras por minuto del movimiento alternativo de traslación. Con estos principios de funcionamiento, el PEULA permite emular, el movimiento alternativo lineal presente en la barra pulida y el comportamiento de cargas de ascenso y descenso, de una unidad de bombeo mecánico.

En la corredera del PEULA (figuras 3 y 4), se instaló una celda de carga tipo $\mathrm{S}$ y un acelerómetro MEMS, permitiendo obtener de manera instantánea la carga y la aceleración. Un posterior tratamiento permite obtener el dinagrama de superficie.

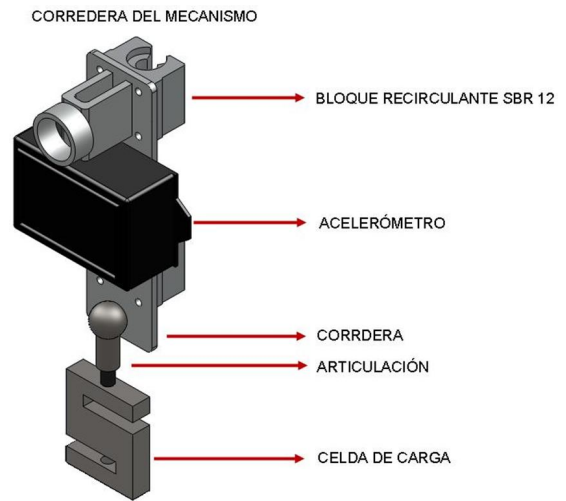

Fig. 4. Corredera del mecanismo.

\subsection{Acelerómetro}

El MMA7361L (Freescale (FM $^{T M}$ utilizado es un sensor de tecnología MEMS, que posee: 3 ejes (XYZ) de aceleración, salidas de tensión análogas, sensibilidad ajustable $( \pm 1.5 \mathrm{~g} \quad \mathrm{o} \quad \pm 6 \mathrm{~g})$, alta sensibilidad (800 mV/g @ 1.5g), bajo voltaje de operación (2.2 a $3.6 \mathrm{~V})$, un filtro pasa bajos de un polo, compensación de temperatura, detección interna de $0 \mathrm{~g}$ y g-, bajo consumo de corriente (400 $\mu \mathrm{A})$ y modo "sleep" $(3 \mu \mathrm{A})$ ideal para manejo con baterías. El acelerómetro MMA7361L consiste de una célula sensible capacitiva ( $g$-cell) y un acondicionador de señal ASIC contenidos dentro del mismo paquete. El g-cell es una estructura mecánica formada de materiales semiconductores (polisilicio). Se puede modelar como una serie de barras unidas a una masa movible central que se desplaza entre las barras fijas. Las barras móviles pueden ser desviadas de su posición de equilibrio cuando se somete el sistema a una aceleración. Las barras g-cell forman dos capacitores "back-toback", es decir, las caras positivas o negativas, hacia adentro. A medida que la barra del medio se mueve con aceleración, la distancia entre las barras cambia, así como también lo hace su capacitancia. El circuito integrado usa técnicas para medir los capacitores $g$-cell y extraer los datos de aceleración de la diferencia entre ambas capacitancias.

\subsection{Celda de carga}

Para medir la carga, se utilizó una celda de carga marca Lexus, tipo S (Tensión), modelo SM, referencia SP06-AL, con las siguientes especificaciones: precisión $\mathrm{C} 3$, sensibilidad de 2 $\mathrm{mV} / \mathrm{V}$, protección IP65, capacidad de $10 \mathrm{Kg}$., impedancia de entrada de $355 \mathrm{Ohm}$, impedancia de salida de 350 Ohm. La celda de carga se instaló en la corredera del mecanismo y lleva consigo un peso fijo, en su carrera de ascenso y descenso, que 
emula la carga que representa la sarta de varillas. Asimismo, ella levanta por medio de un electroimán un segundo peso en su carrera de ascenso que emula el peso del crudo que se está levantando.

\subsection{Sistema de adquisición de datos}

Se desarrolló una arquitectura de adquisición de datos basada en PLC. Como núcleo del sistema se utilizó la CPU 315F-2 PN/DP de Siemens.

Para adquirir la señal proveniente del acelerómetro, se utilizó el amplificador operacional de referencia LF353 y su señal amplificada alimenta a un módulo analógico del PLC. El amplificador se instaló con configuración tipo seguidor de voltaje, para poder adaptar impedancias, es decir, conectar un dispositivo de alta impedancia como el acelerómetro, a un dispositivo de baja impedancia como el módulo analógico del PLC. Se utilizó el módulo analógico SM334 de Siemens.

Para adquirir la señal proveniente de la celda de carga, se utilizó un módulo SIWAREX U de Siemens. El SIWAREX $U$ es un módulo especializado en mediciones de carga (peso) y dinamometría, posee las siguientes características: $0.05 \%$ de límites de error, una resolución interna de 65535 y 50 mediciones /segundo.

\section{OBTENCIÓN DE LA POSICIÓN A PARTIR DE LA SEÑAL DEL ACELERÓMETRO}

La posición instantánea de la barra pulida es obtenida a partir de las mediciones del acelerómetro, el cual debe ser previamente calibrado. La señal de aceleración debe ser integrada dos veces, para obtener la posición instantánea (Seifert, 2007). Se desarrolló un procedimiento de tratamiento de señal, con el fin de obtener los datos con el menor error posible.

\subsection{Calibración del acelerómetro}

Realizando mediciones de los 3 ejes se puede encontrar fácilmente la línea de calibración de cada eje, pues se sabe que teniendo el acelerómetro perfectamente ubicado en un plano $\mathrm{Z}$, las mediciones en cada eje son: $\mathrm{X}=0 \mathrm{~g}, \mathrm{Y}=0 \mathrm{~g}$ y $\mathrm{Z}=$ 1g. En concordancia con las especificaciones del acelerometro, se tiene que $0 \mathrm{~g} \sim 1.45 \mathrm{~V}$ y $1 \mathrm{~g} \sim 2.35$ V. Mediante el promedio de una serie de muestras para cada eje, se determinó la ecuación del acelerómetro.

\subsection{Corrección del "DC OFFSET"}

Las mediciones de aceleración se ven afectadas por la fuerza gravitacional, obteniendo mediciones de $1 \mathrm{~g}$ cuando el dispositivo aún no se ve sometido a ninguna aceleración. De no corregirse el desfase, al integrar la señal se estará integrando una constante, generando errores en la medición (Tuck, 2009). La aceleración en la barra pulida del emulador, corresponde a una señal continua de forma similar a una sinusoide. Si se obtiene una señal que es una función continúa de la aceleración (señal muestreada) y se resta el DC OFFSET del acelerómetro, se obtendrá la señal de aceleración real. Para cada ciclo (carrera/stroke) del emulador (PEULA) se realiza la corrección y se obtiene la aceleración real. Ver figura 5.

\subsection{Adquisición de la aceleración}

Calibrado el sensor y corregido su Offset, se procedió a adquirir la señal de aceleración a una rata de muestreo de $30 \mathrm{~Hz}$ (30.3 milisegundos/ muestra) y 40 segundos para la ventana de muestreo, generando con ello 1200 datos.


Fig. 5. Aceleración sin y con corrección del "DC OFFSET".

\subsection{Marcas de ciclo de aceleración}

Para procesar la información, se requería identificar el inicio y el fin de cada uno de los ciclos del movimiento alternativo de traslación. Se realizó un procedimiento para establecer, a partir de los datos adquiridos, una serie de marcas correspondientes al inicio y fin de cada ciclo de aceleración. Para determinar dichas marcas, es fundamental entender que existen dos cambios en el sentido de la aceleración por ciclo, uno en cada extremo del recorrido de la barra pulida: 
aceleración positiva (+) hacia aceleración negativa (-) / aceleración negativa (-) hacia aceleración positiva (+); los cambios corresponden a los puntos muertos superior e inferior del movimiento de traslación alternativo. Ver figura 6.

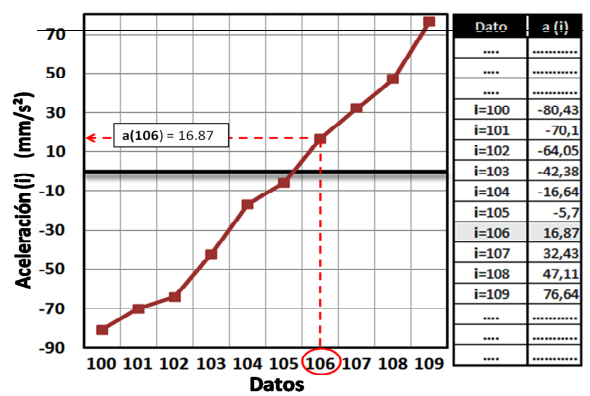

Fig. 6. Cruce por cero de a- hacia a+.

Para establecer las marcas que permitieran determinar cada ciclo de movimiento, se debían identificar entonces los cruces por cero de la aceleración. Se desarrolló un procedimiento para identificar los cruces por cero en el momento de pasar la aceleración de un valor negativo (-) a un valor positivo. La identificación de este punto se realizó mediante una serie de comparaciones de los valores de aceleración respecto al valor cero de referencia. Para determinar los cruces por cero, fueron procesados iterativamente los datos de aceleración, tomando grupos de seis datos por iteración, ver figura 7.

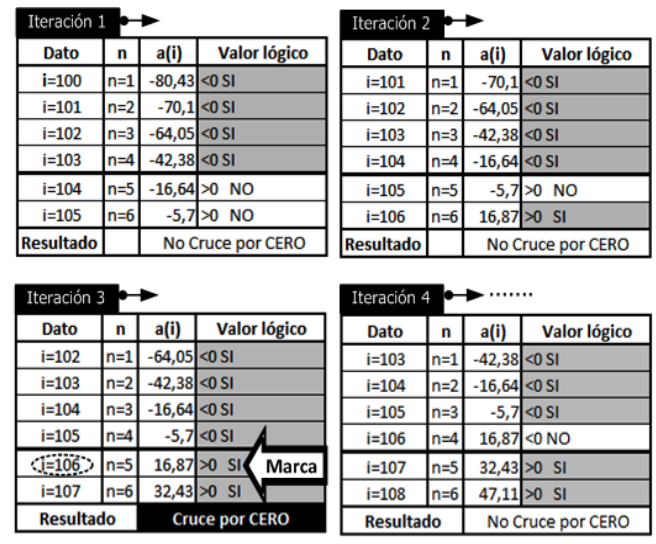

Fig. 7. Iteraciones para determinar el cruce por cero de a-hacia a+.

En una primera iteración el grupo contenía, por ejemplo, los datos desde $i=100$ hasta $i=105$. En la segunda iteración el grupo debía contener ahora, los datos desde $i=101$ hasta $i=106$, o sea un corrimiento de un dato en el grupo, y así sucesivamente. Los datos del grupo, en cada iteración, se procesaban de la siguiente manera: Los cuatro primeros datos $(n=1$ a $n=4)$ se evaluaban si eran menores que cero; los datos cinco y seis $(n=5$ y $n=6)$, se evaluaban si eran mayores que cero. $\mathrm{Si}$ dato $_{\mathrm{n} 1}<0 \wedge$ dato $_{\mathbf{n} 2}<0 \wedge$ dato $_{\mathbf{n} 3}<0 \wedge$ dato $_{\mathbf{n} 4}<0 \wedge$ dato $_{\mathbf{n}}>\mathbf{0} \wedge$ dato $_{\mathbf{n} 6}>\mathbf{0} \rightarrow$ entonces en ésta iteración y en el correspondiente grupo de datos, ocurre el cruce por cero entre los datos $n=4$ y $n=5$; la marca que establece el inicio del ciclo se asocia entonces al dato $n=5$. En el ejemplo mostrado, el cruce se da en la iteración 3 y corresponde al dato $i=106$. Teniendo el número del dato y sabiendo la rata de muestreo de la señal, se obtiene fácilmente el instante (t) en el que ocurre el cruce por cero. La señal de aceleración obtenida y sus respectivas marcas se muestran en la figura 8 .

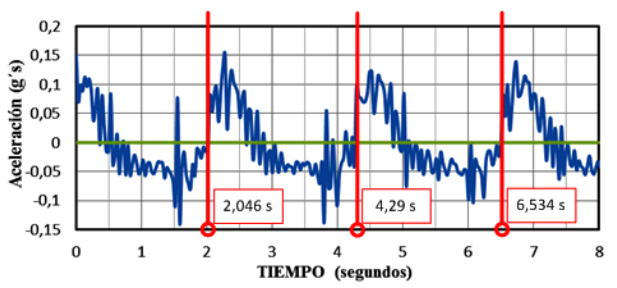

Fig. 8. Marcas de ciclo de aceleración.

\subsection{Ajuste de la aceleración}

Teóricamente, si se consideraran infinitos puntos de datos a lo largo del ciclo, el valor promedio de los datos del ciclo sería cero (0), pero esto normalmente no es posible debido a que la señal de aceleración adquirida puede estar afectada por: ruido electrónico, por el intervalo de muestreo y por vibraciones mecánicas del emulador. Para realizar el ajuste de la aceleración se implementó un filtro basado en una media móvil, donde el valor a ser procesado es el resultado de un promedio de una cierta cantidad de muestras. El ajuste realizado en esta etapa (filtro), consistió en forzar la señal para que el promedio de datos de aceleración a lo largo de un ciclo fuera igual a cero. De no realizar este ajuste, se generaría un error que distorsionaría los datos del proceso en la doble integración que se debe realizar para obtener la posición.

Identificado el inicio y el fin de cada ciclo, se realiza un promedio de los datos de la señal original en el ciclo, y el valor obtenido es restado a cada uno de los datos de la señal original, obteniendo de esta manera una señal "forzada", cuyo promedio sea muy cercano a cero. En la figura 9 se muestra un ciclo de aceleración (original), cuyo promedio es de $-27.9793\left[\mathrm{~mm} / \mathrm{s}^{2}\right]$, al realizar el ajuste se obtiene la nueva curva (forzada) de aceleración con un valor promedio de sólo $0.05\left[\mathrm{~mm} / \mathrm{s}^{2}\right]$. 


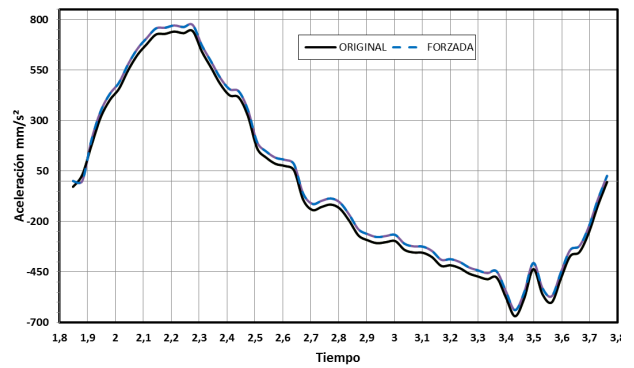

Fig. 9. Ciclos de aceleración original y forzada.

\subsection{Integrar la aceleración para obtener la velocidad.}

Integrar una señal implica encontrar el área bajo la curva de la misma. Dos métodos de integración numérica fueron considerados: sumas de Riemann y aproximaciones trapezoidales, ver figura 10. No se empleó el método de sumas Riemann debido a las pérdidas de muestreo (errores) que podría generar. Se empleó el método de aproximaciones trapezoidales con el fin de obtener una mejor precisión en los datos integrados.

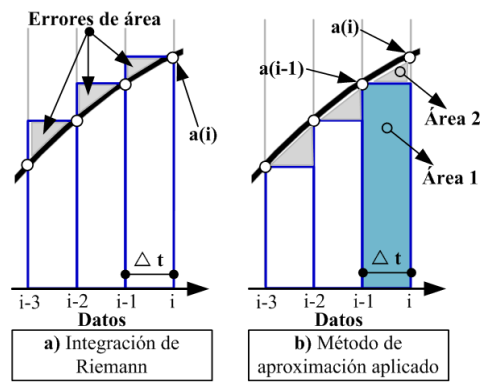

Fig. 10. Métodos numéricos de integración.

El método de sumas de Riemann determina el área bajo la curva por medio de una sumatoria de áreas de rectángulos, cuya base es el $\Delta \mathrm{t}$ entre muestras (30.3 ms), y la altura es el valor de cada muestra $\boldsymbol{a}(\boldsymbol{i})$ de aceleración. El método de aproximaciones trapezoidales, determina el área bajo la curva por medio de una sumatoria de áreas de trapecios (ecuación 1). Para cada trapecio se calculan dos áreas: el área 1 corresponde al área del rectángulo cuya base es el $\Delta \mathrm{t}$ entre muestras $(30.3 \mathrm{~ms})$ y la altura es el valor de la muestra $\boldsymbol{a}(\boldsymbol{i}-1)$ de aceleración; el área dos corresponde al área del triángulo cuya base es el $\Delta \mathrm{t}$ entre muestras (30.3 $\mathrm{ms}$ ) y su altura es la diferencia entre los valores de las muestras $\boldsymbol{a}(\boldsymbol{i})$ y $\boldsymbol{a}(\boldsymbol{i}-1)$ de aceleración. Es evidente que el error producido en el cálculo del área bajo la curva es mucho menor.

$\operatorname{Area}(i)=[a(i-1)] \cdot \Delta t+\left[\frac{a(i)-a(i-1)}{2}\right] \cdot \Delta t$

\subsection{Correcciones a la velocidad}

Una vez calculada la velocidad, se obtiene una gráfica de velocidad contra tiempo, pero dado que al integrar no se contemplaron las condiciones iníciales, ésta se encuentra desplazada en el eje Y. Además, se puede presentar una pequeña pendiente de incremento, la cual se debe a la constante que se genera al integrar la señal en tiempo real, ver fig. 11.



Fig. 11. Velocidad sin corrección.

Para reducir esta rampa de incremento, se realizó una regresión lineal, según el método de los mínimos cuadrados, con los datos almacenados de velocidad.

$$
\begin{aligned}
& m=\frac{\sum x_{i} \cdot y_{i}-n \cdot \bar{X} \cdot \bar{Y}}{\sum x_{i}^{2}-\bar{X}^{2}} \\
& b=\bar{Y}-m \cdot \bar{X} \\
& Y=m \cdot \bar{X}+b \\
& V_{e l} l_{\text {corregida }}=V_{\text {Veriginal }}-Y
\end{aligned}
$$

La línea recta obtenida se restó a los valores de velocidad almacenados, con lo cual se obtuvo la velocidad corregida. Con esta corrección, se desplazó la velocidad al eje horizontal cero y adicionalmente se redujo el error de la pendiente.

\subsection{Marcas de ciclo de velocidad}

Se deben reconocer nuevamente las marcas de comienzo y final de cada ciclo de velocidad. Se aplicó la metodología ya usada para identificar las marcas de aceleración. Los datos utilizados para identificar las marcas de velocidad, fueron los de aceleración, pues estos marcan el verdadero comienzo y final de cada ciclo del sistema.

\subsection{Ajuste de la velocidad}

Al igual que para la aceleración, el promedio del ciclo de velocidad debió ser forzado a cero. El método aplicado fue el mismo: en este caso se tomó un ciclo de velocidad y se calculó su promedio y luego éste se restó de a la velocidad, para forzar al cierre de la curva de velocidad y que 
con ello refleje el comportamiento independiente de cada ciclo de la unidad de bombeo.

Una vez realizados todos estos ajustes y correcciones a los datos de aceleración, finalmente se obtiene la velocidad, ver figura 12 .



Fig. 12. Velocidad.

\subsection{Integrar para obtener la posición.}

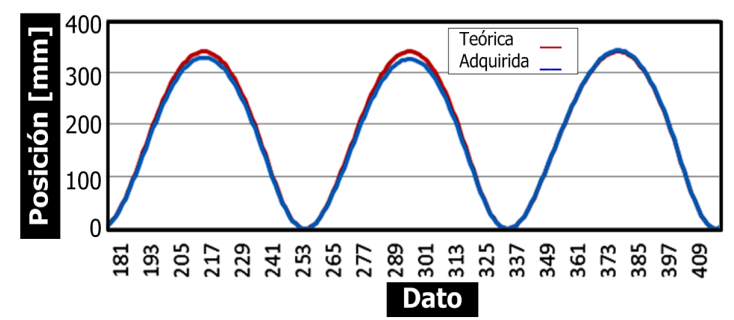

Fig. 13. Posición instantánea obtenida.

Ajustada la velocidad y establecidas sus marcas para inicio y fin de ciclo, debía ahora integrarse para obtener la posición.

Un procedimiento similar al desarrollado para integrar la aceleración, fue utilizado para integrar la velocidad y obtener la posición instantánea del sistema, ver figura 13 .

\section{OBTENCIÓN DE LA CARGA INSTANTÁNEA}

El valor instantáneo de carga presente en el emulador provenía de una medida directa del sensor de carga (celda de carga) conectado al módulo especializado SIWAREX U.

\subsection{Calibración de la celda de carga}

La celda de carga se calibró mediante dos técnicas. La primera, mediante sus valores teóricos dados por el fabricante en la hoja de especificaciones. La segunda es una calibración experimental por medio de pesos calibrados. Capturados los datos se realizó una regresión lineal para obtener la recta de calibración de la celda de carga.

\subsection{Captura de la carga instantánea}

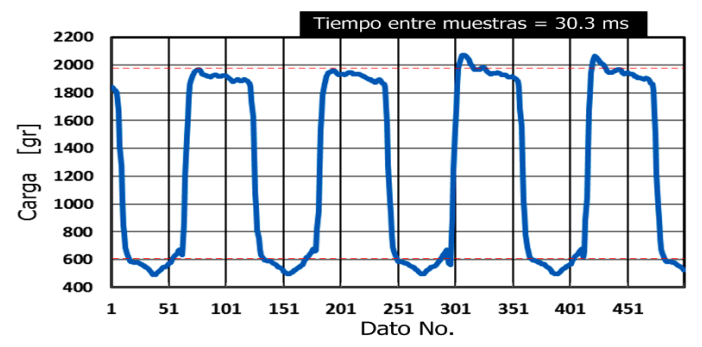

Fig. 14. Carga instantánea capturada.

Para varios ciclos de movimiento alternativo del emulador (PEULA) se capturó el valor de carga instantánea y se contrastó contra los valores esperados. Los pesos instalados en el emulador fueron: peso 1 de 605 gr. (emula la sarta de varillas) y peso 2 de 1190 gr. (emula el peso del crudo). Con esto se tiene que el peso total en ascenso es de 1895 gr. En descenso el peso total es de 605 gr. Debido a que es un sistema móvil y su aceleración fluctúa a lo largo del recorrido se tiene que el peso medido se encuentra en un rango cercano a dichos valores, ver figura 14.

\section{OBTENCIÓN DE LOS DINAGRAMAS}

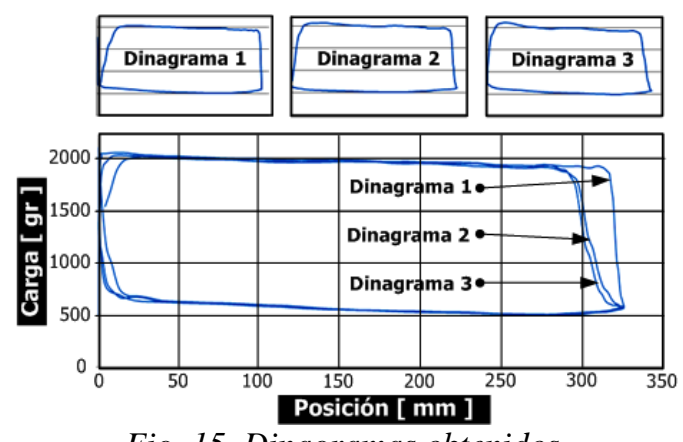

Fig. 15. Dinagramas obtenidos.

Teniendo para cada ciclo, las señales de posición y carga en función del tiempo, lo que procede es relacionarlas entre sí, dado que tienen en común el instante (t) en que fueron capturadas, para obtener una señal (grafica) de carga instantánea contra posición instantánea, por cada ciclo alternativo de movimiento, o sea, el dinagrama de superficie. En cada prueba realizada, se capturaron los datos de aceleración y carga para 3 o 4 ciclos (carreras) de movimiento alternativo, lo cual condujo a que en cada prueba se obtuvieran entre 3 y 4 dinagramas superpuestos. Los dinagramas obtenidos, (figura 15), guardaron absoluta correspondencia con los teóricos esperados. 


\section{RESULTADOS}

La posición obtenida mediante el procesamiento de la señal proveniente del acelerómetro, fue contrastada con el valor teórico esperado, para así obtener el error en su medida. Según la construcción geométrica del PEULA, el desplazamiento máximo correspondía a $340 \mathrm{~mm}$. Se realizaron diecinueve (19) mediciones las cuales contenían entre tres (3) y cuatro (4) ciclos, a diversas velocidades de rotación de la manivela. En total se analizaron 64 ciclos, con los cuales se realizó un análisis estadístico. En la tabla 1, el error promedio fue de $11.49 \mathrm{~mm}(3.38 \%)$, y la frecuencia más alta de resultados se obtuvo en el rango de 0 a $3 \%$ de error.

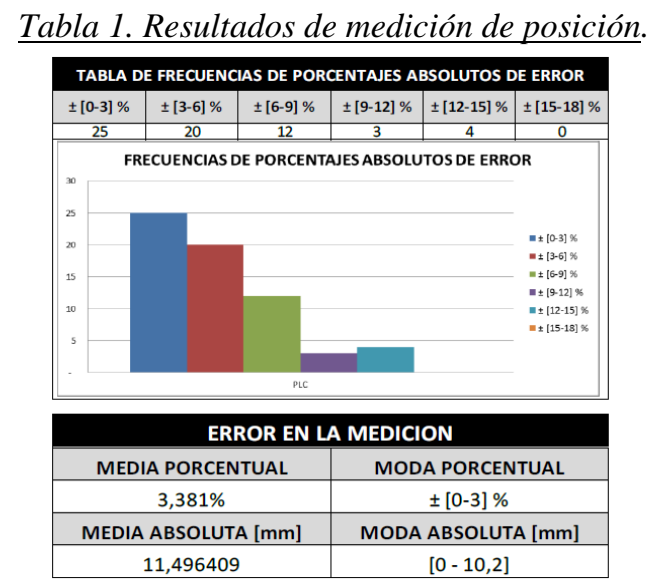

\section{CONCLUSIONES}

Los resultados obtenidos evidencian que mediante el uso de tecnologías MEMS, es posible diseñar y construir una solución de bajo costo, propia e innovadora de hardware y software, que establezca las bases para el concepto de pozo y campo inteligente, destinada a su utilización en campos maduros, a través de la cual se pueda identificar con precisión y rapidez los problemas (fallas) en un pozo, con el fin de disminuir el riesgo operacional y mejorar la producción.

\section{RECONOCIMIENTO}

Este trabajo hace parte del proyecto de investigación 8556 "Desarrollo de un prototipo de pozo inteligente para Campo Escuela Colorado Fase 1", financiado por la Vicerrectoría de Investigación y Extensión de la Universidad Industrial de Santander.

\section{REFERENCIAS}

de Best L. (2012). Shell's Smart Fields - Sustaining and Accelerating Benefits from Intelligent Fields, Intelligent Energy International, Utrecht, 150407-MS SPE Conference Paper.

de Best L. (2006). Smart Fields - Making the Most of Our Assets, SPE Russian Oil and Gas Technical Conference, Moscow (Russia), 103575-MS SPE Conference Paper.

Gao H. (2007). A Literature Review on Smart Well Technology, Production and Operations Symposium, Oklahoma (USA.), 106011-MS SPE Conference Paper.

McCoy J. y Podio L. (1995). Method and Apparatus for measuring pumping rod position and other aspects of a pumping system by use of an accelerometer, Patent US5406482.

Moises G., Rolim T. y Formigli J. (2008). GeDIg: Petrobras Corporate Program for Digital Integrated Field Management, Intelligent Energy Conference and Exhibition, Amsterdam, 112153-MS SPE Conference Paper.

Pari M., N. y Kabir A., H. (2009). Smart wellBenefits, Types of Sensors, Challenges, Economic Consideration, and Application in Fractured Reservoir, SPE Saudia Arabia Section Technical Symposium, 126093-MS SPE Conference Paper.

Sánchez J. y Festini D. (2007). Beam Pumping System Optimization Through Automation, Latin American \& Caribbean Petroleum Engineering Conference, Buenos Aires (Argentina), 108112 SPE Conference Paper.

Seifert K. y Camacho O. (2007). Implementing positioning algorithms using accelerometers. Freescale Semiconductor, Application Note, AN3397, Rev 0.

Tuck K. (2009). Implementing Auto-Zero Calibration Technique for Accelerometers, Freescale Semiconductor, Application Note, AN3447, Rev. 0. 